\title{
Hybrid image potential states in molecular overlayers on graphene
}

\author{
Sasfan Arman Wella, ${ }^{1,2}$ Hiroyuki Sawada, ${ }^{1}$ Nana Kawaguchi, ${ }^{3}$ Fahdzi Muttaqien, ${ }^{1}$ Kouji Inagaki, ${ }^{1}$ Ikutaro Hamada, \\ Yoshitada Morikawa, ${ }^{1,4}$ and Yuji Hamamoto ${ }^{1, *}$ \\ ${ }^{1}$ Department of Precision Science and Technology, Graduate School of Engineering, Osaka University, Suita, Osaka 565-0871, Japan \\ ${ }^{2}$ Department of Physics, Faculty of Mathematics and Natural Sciences, Institut Teknologi Bandung, Bandung 40132, Indonesia \\ ${ }^{3}$ Department of Applied Chemistry, Graduate School of Engineering, Osaka University, Suita, Osaka 565-0871, Japan \\ ${ }^{4}$ Research Center for Ultra-Precision Science and Technology, Graduate School of Engineering, Osaka University, Suita, \\ Osaka 565-0871, Japan
}

(Received 13 May 2017; revised manuscript received 18 October 2017; published 10 November 2017)

\begin{abstract}
The structural and electronic properties of naphthalene adsorbed on graphene are studied from first principles using the van der Waals density functional method. It is shown that naphthalene molecules are stabilized by forming a superstructure with the periodicity of $(2 \sqrt{3} \times 2 \sqrt{3})$ and a tilted molecular adsorption geometry on graphene, in good agreement with the scanning tunneling microscopy (STM) experiments on highly oriented pyrolytic graphite. Our results predict that image potential states (IPSs) are induced by intermolecular interaction on the naphthalene overlayer, hybridizing with the IPSs derived from graphene. The resultant hybrid IPSs are characterized by anisotropic effective mass reflecting the molecular structure of naphthalene. By means of STM simulations, we reveal that one of the hybrid IPSs manifests itself as an oval protrusion distinguishable from naphthalene molecular orbitals, which identifies the origin of an experimental STM image previously attributed to the lowest unoccupied molecular orbital of naphthalene.
\end{abstract}

DOI: 10.1103/PhysRevMaterials.1.061001

Introduction. The emergence of image potential states (IPSs) is a universal nature of metal surfaces, characterized by a set of unoccupied states quantized to a Rydberg series analogous to the hydrogen atom [1,2]. IPSs exist even on graphene, an atomically thin two-dimensional material, where the IPSs on the two surfaces hybridize to form a double Rydberg series corresponding to symmetric and antisymmetric states with respect to the graphene sheet $[3,4]$. More exotic IPSs appear on curved graphene, typical examples of which are tubular IPSs extended around carbon nanotubes [5] and superatom molecular orbitals of $\mathrm{C}_{60}$ fullerene [6]. The hybridization of these IPSs results in the interlayer states with a nearly-free-electron feature in a variety of graphitic materials such as graphite [3], multiwalled [7,8] or bundles $[9,10]$ of carbon nanotubes, fullerite solids $[11,12]$, and carbon nanopeapods [13]. In particular, the interlayer states in graphite are believed to be crucial for the superconductivity of graphiteintercalation compounds [14].

In this Rapid Communication, we extend the concept of the IPS-derived interlayer states to molecular adsorption on solid surfaces. From an applicational point of view, it is of particular importance to understand unoccupied states including IPSs for improving the performance of molecularbased electronic devices, such as organic light-emitting diodes. Naphthalene on highly oriented pyrolytic graphite (HOPG) is a prototype system of aromatic molecules physisorbed on a solid surface and has been investigated intensively thus far [15-19]. Recent scanning tunneling microscopy (STM) experiments

\footnotetext{
${ }^{*}$ Corresponding author: hamamoto@prec.eng.osaka-u.ac.jp

Published by the American Physical Society under the terms of the Creative Commons Attribution 4.0 International license. Further distribution of this work must maintain attribution to the author(s) and the published article's title, journal citation, and DOI.
}

have revealed that naphthalene molecules form a well-ordered superstructure with a tilted molecular adsorption geometry at nearly monolayer coverage [17]. More importantly, the results of angle-resolved two-photon photoemission (2PPE) spectroscopy suggest that the lowest IPS (LIPS) behaves almost as a free electron, despite the presence of the naphthalene overlayer [18]. This is explained by assuming that the molecular overlayer is a dielectric medium with uniform permittivity and only shifts the Rydberg series with the effective mass $m^{*}$ unchanged from the electronic mass $m_{e}$ [1]. However, the well-known Kronig-Penny theory [20] suggests an increase in $m^{*}$ if naphthalene acts as a periodic potential for the IPSs.

To develop a coherent picture of the IPSs at organic-solid interfaces, we here perform first-principles calculations of a simplified model composed of naphthalene on graphene with the van der Waals density functional (vdW-DF) method $[21,22]$. Our results predict that the interaction between naphthalene molecules not only stabilizes the naphthalene superstructure on graphene, but also induces extended states analogous to IPSs on the naphthalene overlayer. The IPSlike states hybridize with the graphene IPSs and play an essential role in the formation of peculiar interface states with
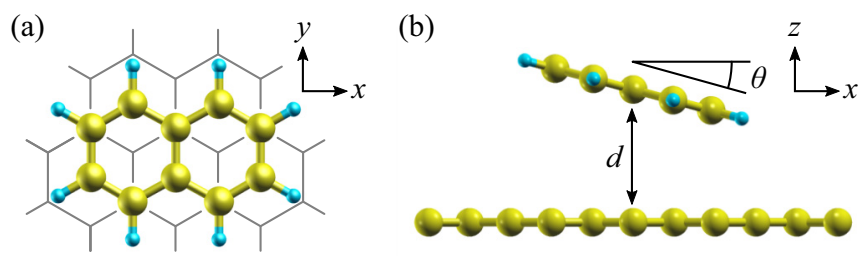

FIG. 1. Schematic views of naphthalene adsorption on graphene. In the top view (a), graphene is represented with its skeleton. In the front view (b), $\theta$ and $d$ denote the tilt angle and distance, respectively, between naphthalene and graphene. 

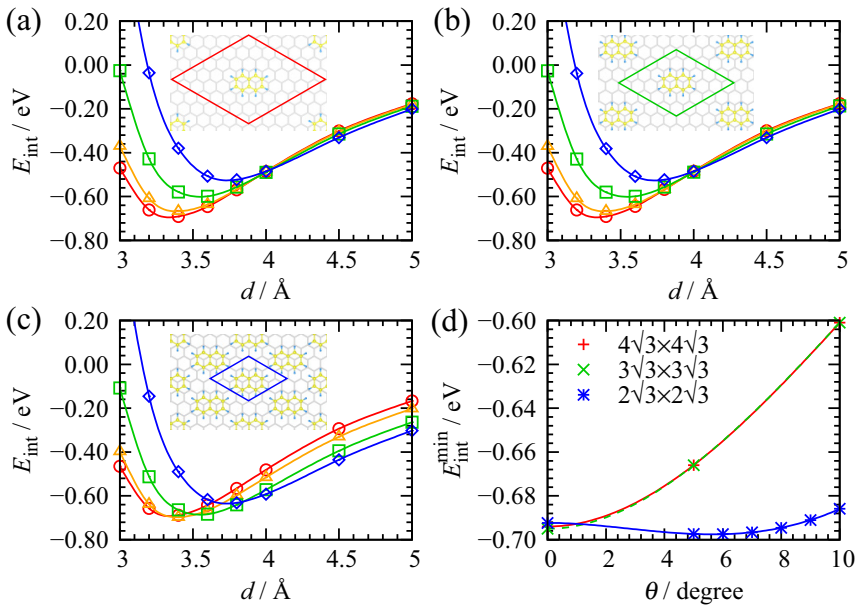

FIG. 2. Interaction energies between naphthalene and graphene. In panels (a), (b) and (c), energy profiles at several tilt angles are plotted as functions of naphthalene-graphene distance for the $(4 \sqrt{3} \times$ $4 \sqrt{3}),(3 \sqrt{3} \times 3 \sqrt{3})$, and $(2 \sqrt{3} \times 2 \sqrt{3})$ unit cells, respectively. Circles $(\circ)$, triangles $(\triangle)$, squares $(\square)$, and diamonds $(\diamond)$ correspond to tilt angles $\theta=0^{\circ}, 5^{\circ}, 10^{\circ}$, and $15^{\circ}$, respectively. In panel (d), the energy minima extracted from panels (a)-(c) are plotted as a function of the tilt angle.

anisotropic $m^{*}$ in naphthalene on graphene. Here we stress that low-lying IPSs are well described by vdW-DF owing to the inclusion of nonlocal correlation [23].

Methods. The calculations are carried out using the STATE code [24] with the norm-conserving pseudopotentials [25]. The plane-wave basis set is used to expand wave functions (charge density) with cutoff energy of 64 Ry (400 Ry). To investigate the coverage dependence of naphthalene on graphene, we adopt three unit cells of graphene with the periodicity of $(2 \sqrt{3} \times 2 \sqrt{3}),(3 \sqrt{3} \times 3 \sqrt{3})$, and $(4 \sqrt{3} \times 4 \sqrt{3})$. Correspondingly, $6 \times 6 \times 1,4 \times 4 \times 1$, and $3 \times 3 \times 1 k$ points are sampled in the Brillouin zone, respectively. The dispersion forces are included through the self-consistent [26-29] vdWDF method [21,22] with the rev-vdW-DF2 functional [30-35]. The lattice constant of graphene obtained with the functional is $2.46 \AA$, in good agreement with experiments [36]. As a reference system, the geometry of an isolated naphthalene molecule is relaxed until the atomic forces fall below $0.08 \mathrm{nN}$ $\left(5.14 \times 10^{-2} \mathrm{eV} / \AA\right)$. A naphthalene molecule thus obtained is $\mathrm{AB}$ stacked on each unit cell of graphene, as shown in Fig. 1. To suppress unphysical interactions between the neighboring cells, a vacuum layer of $\simeq 26 \AA$ is considered. The constant-current STM image of the system is simulated with a density threshold of $6.76 \times 10^{-5} \AA^{-3}$ based on the Tersoff-Hamann method [37,38].

Tilted naphthalene on graphene. To see the role of intermolecular interaction, we first examine the adsorption structure of naphthalene on graphene in three coverage regimes corresponding to the unit cells mentioned above. Stable adsorption structures are explored by varying the distance $d$ and tilt angle $\theta$ between naphthalene and graphene (see Fig. 1) with each structure unchanged. We here ignore the tilting along the short axis of naphthalene, since its effect turns out much smaller than along the long axis. The interaction energy between naphthalene and graphene is calculated as

$$
E_{\text {int }}=E_{\text {nap } / \text { gra }}-E_{\text {nap }}-E_{\text {gra }},
$$

where $E_{\text {nap/gra }}, E_{\text {nap }}$, and $E_{\text {gra }}$ are the total energies of naphthalene on graphene, the isolated naphthalene molecule, and pristine graphene, respectively. In Figs. 2(a)-2(c), the interaction energy (1) is plotted as a function of naphthalenegraphene distance for several tilt angles. The minimal energy $E_{\text {int }}^{\text {min }}$ is extracted from each energy profile by cubic-spline fitting and plotted as a function of $\theta$ as shown in Fig. 2(d). For the $(3 \sqrt{3} \times 3 \sqrt{3})$ and $(4 \sqrt{3} \times 4 \sqrt{3})$ unit cells, $E_{\text {int }}^{\min }$ has a unique minimum at $\theta=0^{\circ}$, i.e., naphthalene tends to be parallel to graphene at low coverage, which is consistent with the assumption adopted in the previous calculations with large unit cells [33,39]. On the other hand, the dispersion force between the molecules works attractively for the $(2 \sqrt{3} \times 2 \sqrt{3})$ unit cell, while the parallel geometry is destabilized by Pauli repulsion. As a result $E_{\text {int }}^{\min }$ displays lower minima at $\theta \simeq 6^{\circ}$, which is roughly $5 \mathrm{meV}$ more stable than at $\theta=0^{\circ}$, indicating that the molecules are stabilized by forming a $(2 \sqrt{3} \times 2 \sqrt{3})$ superstructure with the tilted adsorption geometry. The formation of the naphthalene superstructure is in good agreement with the STM results on HOPG surfaces [17]. It is confirmed that the tilted adsorption structure is insensitive to addition of another graphene layer, or to a perpendicular electric field applied with the effective screening medium method [40,41].

Hybrid image potential states. We next investigate the electronic structure of naphthalene on graphene with a special emphasis on the unoccupied states. To this end, we fully relax the stable structure obtained for the $(2 \sqrt{3} \times 2 \sqrt{3})$ unit cell, although its influence on the adsorption geometry and energy turns out to be negligibly small. We then calculate the energy bands as shown in Fig. 3(a), where the solid (red) and dashed (blue) curves correspond to the band structures along paths $\Gamma \mathrm{M}_{1} \mathrm{~K}_{1} \Gamma$ and $\Gamma \mathrm{M}_{2} \mathrm{~K}_{2} \Gamma$, respectively, in the folded Brillouin zone (see the inset). The difference between the two band structures reflects the fact that the sixfold symmetry of pristine graphene is broken by the adsorption of naphthalene. For clues about the IPSs of the adsorbed system, we here take the LIPS of pristine graphene, the band bottom of which is $2.84 \mathrm{eV}$ from the Fermi level at the $\Gamma$ point [42] (see Fig. $\mathrm{S} 1$ of Supplemental Material [43]). Interestingly, a similar quasiparabolic band can also be found in Fig. 3(a), where the band bottom slightly shifts downwards to $2.79 \mathrm{eV}$. Apparently, the latter is consistent with the 2PPE results of naphthalene on HOPG [18], in which the band dispersion of the LIPS seems unaffected by the molecular adsorption. However, the large amplitudes of the graphene LIPS near the naphthalene adsorption height $d \simeq 3.44 \AA$ suggest possible hybridization with the molecular orbitals (MOs) of naphthalene.

In order to gain insight into the interaction between the graphene LIPS and the naphthalene MOs, we inspect the wave functions of unoccupied states labeled $\mathrm{U}_{1}-\mathrm{U}_{4}$ at the $\Gamma$ point, as shown in the lower panels of Fig. 3. From the comparison with the naphthalene MOs, $\mathrm{U}_{1}\left(\mathrm{U}_{3}\right)$ can be assigned to a state mainly derived from the LUMO+1 (LUMO+2) of naphthalene, where LUMO stands for the lowest unoccupied molecular orbital [44]. Note that the positive (negative) dispersions near $\Gamma$ result from the overlap between adjacent wave functions in an in- (out-of-) phase configuration. In sharp 

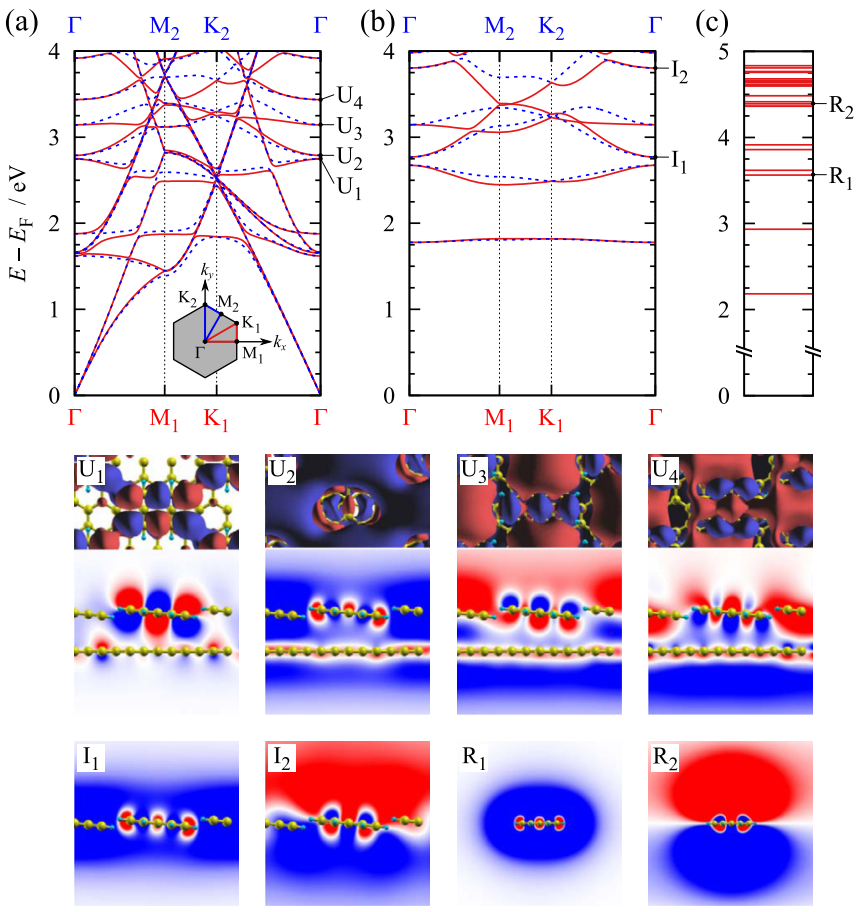

FIG. 3. Band structures above the Fermi level $\left(E_{\mathrm{F}}\right)$ of (a) naphthalene on graphene and (b) the naphthalene monolayer calculated with the $(2 \sqrt{3} \times 2 \sqrt{3})$ unit cell. The energy levels of the isolated naphthalene molecule is shown in panel (c) for comparison. In panels (b) and (c), the origin of energy is determined so that the vacuum level coincides with that in the adsorbed system (a). The solid (red) and dashed (blue) curves denote the energy dispersions along paths $\Gamma \mathrm{M}_{1} \mathrm{~K}_{1} \Gamma$ and $\Gamma \mathrm{M}_{2} \mathrm{~K}_{2} \Gamma$, respectively, in the folded Brillouin zone shown in the inset of panel (a). In the lower panels, the top (front) views of the wave functions for several unoccupied states are represented by isosurfaces (color plots). We show only the real parts of the wave functions, where the red and blue colors correspond to, e.g., positive and negative amplitudes, respectively.

contrast to the localized MO-like states, the wave functions of $\mathrm{U}_{2}$ and $\mathrm{U}_{4}$ are extended both around naphthalene and graphene. Thus these states are no longer simple graphene IPSs, despite the presence of the quasiparabolic dispersions. Rather, the extended behavior of $\mathrm{U}_{2}$ recalls interlayer states realized between graphene LIPSs [3], suggesting that the naphthalene overlayer mimics graphene in the formation of $\mathrm{U}_{2}$.

To identify the origin of the peculiar nature of $U_{2}$, we calculate the energy bands of the naphthalene monolayer without graphene as shown in Fig. 3(b). Here the geometry of naphthalene is fixed to the adsorption structure in the $(2 \sqrt{3} \times 2 \sqrt{3})$ unit cell. One readily notices that there appear quasiparabolic dispersions with band minima of 2.77 and $3.80 \mathrm{eV}$ at $\Gamma$, which are analogous to the two lowest IPSs of graphene. The similarity becomes even clearer from the wave functions of the IPS-like state labeled $\mathrm{I}_{1}\left(\mathrm{I}_{2}\right)$ in Fig. 3(b), which is roughly symmetric (antisymmetric) with respect to the naphthalene layer, corresponding to the LIPS of graphene with even (odd) parity [4]. Unlike the isotropic effective mass of the graphene IPSs $m^{*} / m_{e}=1.02$, the IPS-like states of the naphthalene layer exhibit a strong anisotropy in $m^{*}$ as
TABLE I. Effective masses of the IPS-like states in naphthalene on graphene and the naphthalene monolayer estimated from Fig. 3. The results are obtained in four directions from the $\Gamma$ point in the Brillouin zone.

\begin{tabular}{lcccc}
\hline \hline & $\Gamma \rightarrow \mathrm{M}_{1}$ & $\Gamma \rightarrow \mathrm{K}_{1}$ & $\Gamma \rightarrow \mathrm{M}_{2}$ & $\Gamma \rightarrow \mathrm{K}_{2}$ \\
\hline $\mathrm{U}_{2}$ & 1.00 & 1.07 & 1.27 & 1.43 \\
$\mathrm{U}_{4}$ & 3.13 & 2.70 & 2.10 & 1.89 \\
$\mathrm{I}_{1}$ & 0.98 & 1.05 & 1.23 & 1.36 \\
$\mathrm{I}_{2}$ & 1.90 & 1.97 & 2.13 & 2.23 \\
\hline \hline
\end{tabular}

shown in Table I. For example, $I_{1}$ behaves like the graphene LIPS along the long axis of naphthalene, while it gets heavier along the short axis. $\mathrm{I}_{2}$ also shows a similar anisotropy but is nearly twice heavier than $\mathrm{I}_{1}$. As the intermolecular separation increases, the IPS-like states lose the energy dispersions and convert to the Rydberg states [45] of the isolated naphthalene molecule labeled $\mathrm{R}_{1}$ and $\mathrm{R}_{2}$ in Fig. 3(c). From this, it is clear that the odd (even) parity of $\mathrm{I}_{1}\left(\mathrm{I}_{2}\right)$ originates from the $s-(p-)$ like orbital of $\mathrm{R}_{1}\left(\mathrm{R}_{2}\right)$.

We also examine how the wave functions evolve as the naphthalene layer approaches graphene. As the naphthalenegraphene distance is decreased, $\mathrm{I}_{1}$ starts to hybridize with the graphene LIPS with even parity, forming bonding and antibonding states. The bonding state is initially stabilized by the hybridization, while it is destabilized by Pauli repulsion near the adsorption distance. As a result of the cancellation, the energy of the bonding state $U_{2}$ remains close to that of the graphene LIPS (Fig. S1), as seen in Fig. 3(a). From Table I, one notices that $\mathrm{U}_{2}$ retains the anisotropic $m^{*}$ of $\mathrm{I}_{1}$ even after the hybridization. On the other hand, the antibonding state between $\mathrm{I}_{1}$ and the graphene LIPS is destabilized monotonically as the naphthalene-graphene distance is decreased. As a result, the antibonding state hybridizes with the naphthalene LUMO+2 to form $\mathrm{U}_{4}$, hence the signature of the $\mathrm{MO}$ in the corresponding wave function. More importantly, $\mathrm{U}_{4}$ displays an inverse anisotropy in $m^{*}$ as compared with $\mathrm{U}_{2}$, which derives from the hybridization between adjacent LUMO+2 states along the short axis of naphthalene. We also note that the parabolic band above $\mathrm{U}_{4}$ derives from the bonding hybridization between $\mathrm{I}_{2}$ and the graphene LIPS with odd parity. Further details about the evolution of these unoccupied states are shown in Fig. S2 of the Supplemental Material [43].

STM simulation. Finally, we simulate the STM images of naphthalene on graphene using the relaxed geometry and charge density obtained for the $(2 \sqrt{3} \times 2 \sqrt{3})$ unit cell. For simplicity, we neglect the shift in the energy bands due to the electric field from the tip. The simulated STM images are obtained at several sample bias voltages $V_{\mathrm{S}}$ as shown in Fig. 4 , where the results at negative (positive) $V_{\mathrm{S}}$ probe occupied (unoccupied) states. The image at $V_{\mathrm{S}}=-1 \mathrm{~V}$ is characterized by four bright spots on each naphthalene molecule, which are asymmetric with respect to the long axis of naphthalene. The bright spots correspond to the carbon atoms of naphthalene not stacking with those of graphene in analogy to HOPG surfaces [46], detecting the hybridization between the highest occupied molecular orbital (HOMO) of naphthalene and the $\pi$ bands of graphene. The contribution from the HOMO becomes 

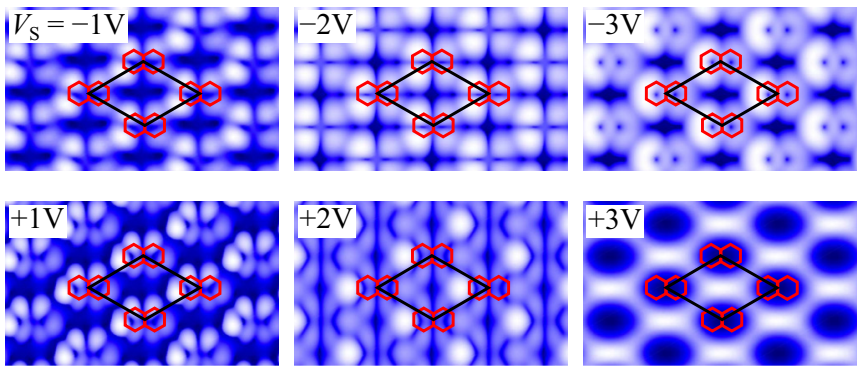

HOMO-2 HOMO-1

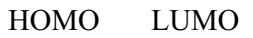

$\mathrm{LUMO}+1 \mathrm{LUMO}+2$

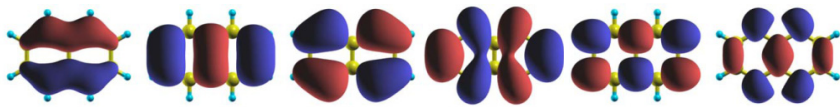

FIG. 4. Simulated STM images of naphthalene on graphene with the $(2 \sqrt{3} \times 2 \sqrt{3})$ periodicity. The results are obtained at sample bias voltages $V_{\mathrm{S}}= \pm 1, \pm 2$, and $\pm 3 \mathrm{~V}$. The bright (dark) regions correspond to protrusions (depressions), and the rhombus and hexagons denote the $(2 \sqrt{3} \times 2 \sqrt{3})$ unit cell and the benzene rings of naphthalene, respectively. Several MOs of naphthalene are depicted for comparison in the lower panels.

more prominent at $V_{\mathrm{S}}=-2 \mathrm{~V}$, where neighboring bright spots merge into dumbbell-like protrusions with two orientations, which is similar to the experimental image at $V_{\mathrm{S}}=-3.1 \mathrm{~V}$ [17] in that the pattern consists of two types of bright spots. At $V_{\mathrm{S}}=-3 \mathrm{~V}$, the contribution from the HOMO -1 state is superposed, and as a result the obtained image resembles the benzene rings of naphthalene molecules.

At positive sample biases, on the other hand, the simulated STM images reflect the characteristics of unoccupied states including the IPS-like states. As in the case of $V_{\mathrm{S}}=-1 \mathrm{~V}$, the absence of the mirror symmetry is also seen at $V_{\mathrm{S}}=+1$ $\mathrm{V}$, where the $\pi$ orbitals of graphene now hybridize with the LUMO of naphthalene. At $V_{\mathrm{S}}=+2 \mathrm{~V}$, protrusions analogous to the naphthalene LUMO merge with those of neighboring molecules, and the bright spots corresponding to higher carbon atoms form a triangular lattice. Intriguingly, the STM image obtained at $V_{\mathrm{S}}=+3 \mathrm{~V}$ displays oval protrusions at the interstitial regions between the molecules, which are quite unlike any naphthalene MOs. This indicates that the hybrid IPS $\left(\mathrm{U}_{2}\right)$ predominantly contributes to the STM image. Our results explain the origin of the elongated oval protrusions observed experimentally, which have previously been attributed to the LUMO of naphthalene [17]. This also suggests that the hybrid IPSs can be imaged experimentally with STM.

Conclusions. We have theoretically investigated the naphthalene adsorption on graphene using the vdW-DF method. Our results show that the molecular adsorption is stabilized by the formation of the $(2 \sqrt{3} \times 2 \sqrt{3})$ superstructure with a tilted adsorption geometry, in good agreement with the STM results on HOPG surfaces. More importantly, the intermolecular interaction induces IPS-like states on the naphthalene overlayer, which hybridize with the graphene IPSs in the bonding and antibonding manners. The impact of the naphthalene adsorption appears most prominently in the anisotropic effective mass of the resultant hybrid IPSs, which strongly reflects the molecular structure of naphthalene. It is expected that similar hybrid IPSs widely exist at interfaces of molecular overlayers and solid surfaces.

Acknowledgments. We thank Professors Toshiaki Munakata and Takashi Yamada for valuable discussions. S.A.W. acknowledges financial support by the Marubun Research Promotion Foundation. The present study was partly supported by Grants-in Aid for Scientific Research on Innovative Areas "3D Active-Site Science" (Grants No. JP26105010 and No. JP26105011) and "Molecular Architectonics" (Grant No. JP25110006); Grant-in-Aid for Young Scientists (B) (Grant No. JP15K17682) from the Japan Society for the Promotion of Science (JSPS); the Advanced-Catalytic-Transformation program for Carbon utilization (ACT-C) of Japan Science and Technology Agency (JST), "Elements Strategy Initiative for Catalysts \& Batteries" (ESICB) supported by the Ministry of Education Culture, Sports, Science and Technology, Japan (MEXT); and the JSPS Core-to-Core Program (Type A) "Advanced Research Networks: Computational Materials Design on Green Energy." The numerical calculations in this work have been done with the facilities of the Supercomputer Center, Institute for Solid State Physics, University of Tokyo.
[1] M. W. Cole and M. H. Cohen, Phys. Rev. Lett. 23, 1238 (1969).

[2] P. M. Echenique and J. B. Pendry, J. Phys. C: Solid State Phys. 11, 2065 (1978).

[3] M. Posternak, A. Baldereschi, A. J. Freeman, E. Wimmer, and M. Weinert, Phys. Rev. Lett. 50, 761 (1983).

[4] V. M. Silkin, J. Zhao, F. Guinea, E. V. Chulkov, P. M. Echenique, and H. Petek, Phys. Rev. B 80, 121408 (2009).

[5] B. E. Granger, P. Král, H. R. Sadeghpour, and M. Shapiro, Phys. Rev. Lett. 89, 135506 (2002).

[6] M. Feng, J. Zhao, and H. Petek, Science 320, 359 (2008).

[7] M. Zamkov, H. S. Chakraborty, A. Habib, N. Woody, U. Thumm, and P. Richard, Phys. Rev. B 70, 115419 (2004).

[8] M. Zamkov, N. Woody, B. Shan, H. S. Chakraborty, Z. Chang, U. Thumm, and P. Richard, Phys. Rev. Lett. 93, 156803 (2004).

[9] A. Thess, R. Lee, P. Nikolaev, H. Dai, P. Petit, J. Robert, C. Xu, Y. H. Lee, S. G. Kim, A. G. Rinzler, D. T. Colbert, G. E.
Scuseria, D. Tománek, J. E. Fischer, and R. E. Smalley, Science 273, 483 (1996).

[10] S. Okada, A. Oshiyama, and S. Saito, Phys. Rev. B 62, 7634 (2000).

[11] S. Saito and A. Oshiyama, Phys. Rev. Lett. 66, 2637 (1991).

[12] J. Zhao, M. Feng, J. Yang, and H. Petek, ACS Nano 3, 853 (2009).

[13] S. Okada, S. Saito, and A. Oshiyama, Phys. Rev. Lett. 86, 3835 (2001).

[14] G. Csányi, P. B. Littlewood, A. H. Nevidomskyy, C. J. Pickard, and B. D. Simons, Nat. Phys. 1, 42 (2005).

[15] C. Bondi, P. Baglioni, and G. Taddei, Chem. Phys. 96, 277 (1985).

[16] U. Bardi, S. Magnanelli, and G. Rovida, Langmuir 3, 159 (1987).

[17] T. Yamada, Y. Takano, M. Isobe, K. Miyakubo, and T. Munakata, Chem. Phys. Lett. 546, 136 (2012). 
[18] T. Yamada, M. Isobe, M. Shibuta, H. S. Kato, and T. Munakata, J. Phys. Chem. C 118, 1035 (2014).

[19] F. Sojka, M. Meissner, T. Yamada, T. Munakata, R. Forker, and T. Fritz, J. Phys. Chem. C 120, 22972 (2016).

[20] G. Grosso and G. P. Parravicini, Solid State Physics (Academic Press, New York, 2000).

[21] M. Dion, H. Rydberg, E. Schröder, D. C. Langreth, and B. I. Lundqvist, Phys. Rev. Lett. 92, 246401 (2004).

[22] K. Berland, V. R. Cooper, K. Lee, E. Schröder, T. Thonhauser, P. Hyldgaard, and B. I. Lundqvist, Rep. Prog. Phys. 78, 066501 (2015).

[23] I. Hamada, Y. Hamamoto, and Y. Morikawa, J. Chem. Phys. 147, 044708 (2017).

[24] Y. Morikawa, K. Iwata, and K. Terakura, Appl. Surf. Sci. 169170, 11 (2001).

[25] N. Troullier and J. L. Martins, Phys. Rev. B 43, 1993 (1991).

[26] T. Thonhauser, V. R. Cooper, S. Li, A. Puzder, P. Hyldgaard, and D. C. Langreth, Phys. Rev. B 76, 125112 (2007).

[27] G. Román-Pérez and J. M. Soler, Phys. Rev. Lett. 103, 096102 (2009).

[28] J. Wu and F. Gygi, J. Chem. Phys. 136, 224107 (2012).

[29] Y. Hamamoto, I. Hamada, K. Inagaki, and Y. Morikawa, Phys. Rev. B 93, 245440 (2016).

[30] I. Hamada, Phys. Rev. B 89, 121103 (2014).

[31] I. Lončarić and V. Despoja, Phys. Rev. B 90, 075414 (2014).

[32] P. Han, K. Akagi, F. Federici Canova, H. Mutoh, S. Shiraki, K. Iwaya, P. S. Weiss, N. Asao, and T. Hitosugi, ACS Nano 8, 9181 (2014).

[33] F. Huttmann, A. J. Martínez-Galera, V. Caciuc, N. Atodiresei, S. Schumacher, S. Standop, I. Hamada, T. O. Wehling, S. Blügel, and T. Michely, Phys. Rev. Lett. 115, 236101 (2015).
[34] B. Yang, J. Björk, H. Lin, X. Zhang, H. Zhang, Y. Li, J. Fan, Q. Li, and L. Chi, J. Am. Chem. Soc. 137, 4904 (2015).

[35] M. Callsen and I. Hamada, Phys. Rev. B 91, 195103 (2015).

[36] Y. Baskin and L. Meyer, Phys. Rev. 100, 544 (1955).

[37] J. Tersoff and D. R. Hamann, Phys. Rev. B 31, 805 (1985).

[38] J. Tersoff and D. R. Hamann, Phys. Rev. Lett. 50, 1998 (1983).

[39] S. D. Chakarova-Käck, E. Schröder, B. I. Lundqvist, and D. C. Langreth, Phys. Rev. Lett. 96, 146107 (2006).

[40] M. Otani and O. Sugino, Phys. Rev. B 73, 115407 (2006).

[41] I. Hamada, M. Otani, O. Sugino, and Y. Morikawa, Phys. Rev. B 80, 165411 (2009).

[42] Recently we have found that vdW-DF reproduces the low-lying IPS levels of graphene obtained using the LDA+image potential correction $[4,23]$.

[43] See Supplemental Material at http://link.aps.org/supplemental/ 10.1103/PhysRevMaterials.1.061001 for additional information about the unoccupied states of the present system.

[44] Density functional theory in general underestimates the LUMO level, while it also lacks dynamical surface polarization [47-49]. This cancellation enables one to describe the LUMO level of molecules on solid surfaces reasonably well .

[45] A. B. F. Duncan, Rydberg Series in Atoms and Molecules (Academic Press, New York, 1971).

[46] D. Tománek, S. G. Louie, H. J. Mamin, D. W. Abraham, R. E. Thomson, E. Ganz, and J. Clarke, Phys. Rev. B 35, 7790 (1987).

[47] J. B. Neaton, M. S. Hybertsen, and S. G. Louie, Phys. Rev. Lett. 97, 216405 (2006).

[48] K. S. Thygesen and A. Rubio, Phys. Rev. Lett. 102, 046802 (2009).

[49] J. M. Garcia-Lastra, C. Rostgaard, A. Rubio, and K. S. Thygesen, Phys. Rev. B 80, 245427 (2009). 